\title{
Dual-modulation, dual-wavelength, optical polarimetry system for glucose monitoring
}

Zhen Fang Yu

Casey W. Pirnstill

Gerard L. Coté 


\title{
Dual-modulation, dual-wavelength, optical polarimetry system for glucose monitoring
}

\author{
Zhen Fang Yu, ${ }^{\mathrm{a}, \mathrm{b}}$ Casey W. Pirnstill, ${ }^{\mathrm{b}, \mathrm{c}}$ and Gerard L. Cotée, \\ aniversity of Electronic Science and Technology of China, School of Optoelectronic Information, No. 4, Section 2 , \\ North Jianshe Road, Chengdu 610054, China \\ ${ }^{\mathrm{b} T}$ Texas A\&M University, Department of Biomedical Engineering, 5045 Emerging Technologies, Building 3120 TAMU, \\ College Station, Texas 77843-3120, United States \\ 'Wright-Patterson Air Force Base, 711th Human Performance Wing, Human Effectiveness Directorate, Warfighter Interface Division, \\ Applied Neuroscience Branch, WPAFB, 2610 Seventh Street, Building 441, Ohio 45433, United States \\ dTexas A\&M University Experiment Station, Center for Remote Health Technologies and Systems, MS3120, \\ Emerging Technologies Building, College Station, Texas 77843, United States
}

\begin{abstract}
A dual modulation optical polarimetry system utilizing both laser intensity and polarization modulation was designed, built, and tested. The system was designed to reduce complexity and enhance the speed in order to facilitate the reduction of motion-induced time-varying birefringence, which is one of the major limitations to the realization of polarimetry for glucose monitoring in the eye. The high-speed less complex technique was tested using in vitro phantom studies with and without motion artifact introduced. The glucose concentration ranged from 0 to $600 \mathrm{mg} / \mathrm{dl}$ and the glucose measurements demonstrated a standard error of prediction to within $8.1 \mathrm{mg} / \mathrm{dl}$ without motion and to within $13.9 \mathrm{mg} / \mathrm{dl}$ with motion. Our feedback control systems took less than $10 \mathrm{~ms}$ to reach stabilization, which is adequately fast to eliminate the effect of time-varying birefringence. The results indicate that this new optical polarimetric approach has improved the speed and reduced the complexity, showing the potential for it to be used for noninvasive glucose measurements. $\odot 2016$ Society of Photo-Optical Instrumentation Engineers (SPIE) [DOI: 10.1117/1.JBO.21.8.087001]
\end{abstract}

Keywords: optical activity; polarimetry; glucose monitoring; noninvasive; motion artifact.

Paper 160156PRR received Mar. 10, 2016; accepted for publication Jul. 8, 2016; published online Aug. 1, 2016.

\section{Introduction}

Diabetes afflicts an estimated 347 million people worldwide, and nearly 29.1 million people in the United States. ${ }^{1,2}$ Intensive management of blood sugars is an effective way to prevent or at least slow the progression of diabetic complications and this management requires frequent monitoring of glucose levels followed by mitigation via insulin or lifestyle changes. ${ }^{3}$ Currently, the most common commercial methods for glucose monitoring are invasive and require a blood sample from the patients' finger or forearm each time a reading is needed. In some cases, patients use continuous glucose monitoring (CGM) approaches, which require an indwelling probe, typically inserted in the abdomen. Although the CGM approaches provide continuous data, they require frequent calibration; the probes need to be replaced typically every 3 and 7 days depending on the brand. ${ }^{4}$ Both of the finger/forearm stick and CGM approaches can be painful, uncomfortable, and can lead to infection for the end user. ${ }^{5}$ Therefore, development of a noninvasive sensor can overcome these problems, greatly enhance the experience for the end user, and potentially facilitate better management of diabetes.

Over the past few decades, many companies and universities have and are attempting to quantify glucose using various noninvasive optical methods, which include, but are not limited to Raman spectroscopy, ${ }^{6,7}$ near-infrared spectroscopy, ${ }^{8,9}$ optical coherence tomography, ${ }^{10-12}$ photoacoustic spectroscopy, ${ }^{13}$ fluorescence, ${ }^{14-16}$ and optical polarimetry. ${ }^{17,18}$ Each of these approaches has their strengths and drawbacks as covered not only in the above articles but also in several good review articles on the topic ${ }^{4,19-22}$ and so we will not go into detail here on each of them but rather focus briefly on the polarimetric approach.

Optical polarimetry applied to glucose detection relies on optical activity or rather chirality of the glucose molecule, which rotates the polarization plane of a linearly polarized light. The amount of rotation is proportional to specific rotation, the concentration of the optically active compound, and the length of the sample. The following equation describes this interaction between an optical active molecule with polarized light, namely

$[\alpha]_{\lambda}=\frac{\alpha}{L C}$

in which $[\alpha]_{\lambda}$ is the specific rotation of the optically active molecule at a given wavelength $(\lambda), \alpha$ is the observed rotation, $C$ is the sample concentration, and $L$ is the sample path length.

Optical polarimetry for glucose monitoring has been thoroughly discussed in the literature. ${ }^{17-32}$ Briefly, polarimetry was first used in the food industry in $1956^{23}$ and was first proposed as a possible method for noninvasive glucose monitoring across the aqueous humor of the eye in $1982 .{ }^{17}$ The advantages that were shown over the years were that the aqueous humor glucose is correlated with blood glucose, it is a clear medium with negligible loss of polarization due to scattering effects, had the submillidegree sensitivity to measure the small rotations, and had the potential to overcome motion artifact. ${ }^{24-31}$ 
A closed-loop dual-wavelength polarimetric system utilizing a real-time feedback controller was designed and used for both in vitro and in vivo rabbit studies. ${ }^{29-31}$ The approach was sufficiently fast enough to show some success in measuring glucose concentrations in the presence of motion-induced birefringence but the overall response time of this system was roughly $300 \mathrm{~ms}$, which was limited by the Faraday modulator's (FM) modulation frequency. A second system was developed using ferrite-based FM to decrease the stabilization time. ${ }^{32}$ Although using the new ferrite FM provided improved system response speed, it still required the more complex two detectors for differentiating the two wavelength signals and suffered from electromagnetic interference and $1 / f$ noise.

To date, these studies have been performed in vitro or using an eye coupling device on anesthetized rabbits with minimal motion and, although the results are promising, by enhancing the system speed and reducing the system complexity, a more effective and efficient system is anticipated that will provide near real time monitoring needed to overcome the noise from time-varying birefringence expected in further in vivo studies.

\section{Materials and Methods}

\subsection{Optical Polarimetry System and In Vitro Test Materials}

The optical configuration of our experiment is shown in Fig. 1 and includes a 635-nm laser diode with maximum power of $7 \mathrm{~mW}$ (Power Technology Inc., Little Rock, Arkansas) as well as a green laser diode with a maximum power of $22 \mathrm{~mW}$ of power at a wavelength of $515 \mathrm{~nm}$ (Oxxius S.A. Lannion, France). Both lasers set to output $3 \mathrm{~mW}$ and the power at the sample was even less and, for any human studies, would be less than the FDA standards for maximum power through the eye. ${ }^{33}$

The lasers were modulated using synchronous sinusoidal signals and linear amplifiers (carrier frequencies: $f_{\mathrm{c} 1}=45 \mathrm{kHz}$, $f_{\mathrm{c} 2}=82 \mathrm{kHz}$ ). The sinusoidal signals were generated from synchronous sine wave generator programmed in LabVIEW 10.0 (National Instruments, Austin, Texas) and implemented through a PCI-6713 card (National Instruments, Austin, Texas). The modulated beams from both sources were linearly polarized in the horizontal direction employing Glan-Thompson
100,000:1 linear polarizers (P) (Newport, Irvine, California). The individual beams propagated through respective in-housebuilt Faraday compensators (FCs) that serve as rotation compensators in order to achieve closed-loop feedback control. These compensators were made of 1-cm-long terbium-gallium-garnet (TGG) crystals (Deltronic Crystal Inc., Dover, New Jersey), which were inside bobbins wrapped with electrical magnetically coated coils of wire. TGG crystal was employed since it provided a high Verdet-constant to get the desired optical rotation for the generated field without losing a significant amount of optical signal transmitted through it.

The two beams were made coincident by a beam splitter/ combiner. The amplitude of each beam was modulated by a ferrite-based FM at frequency of $f_{\mathrm{m}}=8.75 \mathrm{kHz}$. The coil around the ferrite core was powered with an audio amplifier (Radio Shack, Fort Worth, Texas) connected in series with a $0.18-\mu \mathrm{F}$ capacitor in order to achieve resonance at the modulation frequency. The modulation frequency signal was derived from the same synchronous sine wave generator which was used to power the lasers.

Following the ferrite-based FM, the light beam proceeded through a rectangular sample cell (Starna Cells Incorporated, Atascadero California) constructed of birefringent material with a path length of $1 \mathrm{~cm}$. Following the sample was a second Glan-Thompson polarizer, known as the analyzer, which was oriented perpendicular to the initial polarizer in the layout. Since the combined light beams at the two wavelengths each had separate carrier modulated frequencies $\left(f_{\mathrm{c} 1}, f_{\mathrm{c} 2}\right)$, detection could be performed using a single biased photo-diode light detector (Thor Labs, Inc., Newton, New Jersey), which converted the optical signals into electrical signals.

The electrical signals were amplified by a wide-bandwidth transimpedance amplifier (CVI Melles Griot, Albuquerque, New Mexico) and fed into two lock-in amplifiers (Stanford Research Systems, Sunnyvale, California). The DC output voltages from the lock-in amplifiers served as the inputs to proportional-integral-derivative (PID) controllers programmed in LabVIEW (10.0, 32 bit, National Instruments, Austin, Texas) using a field programmable gate array, which provided realtime, closed-loop, feedback. The outputs from PID controllers were used to drive the respective FCs via linear driver circuitry. This circuitry provided sufficient current to each FC to produce

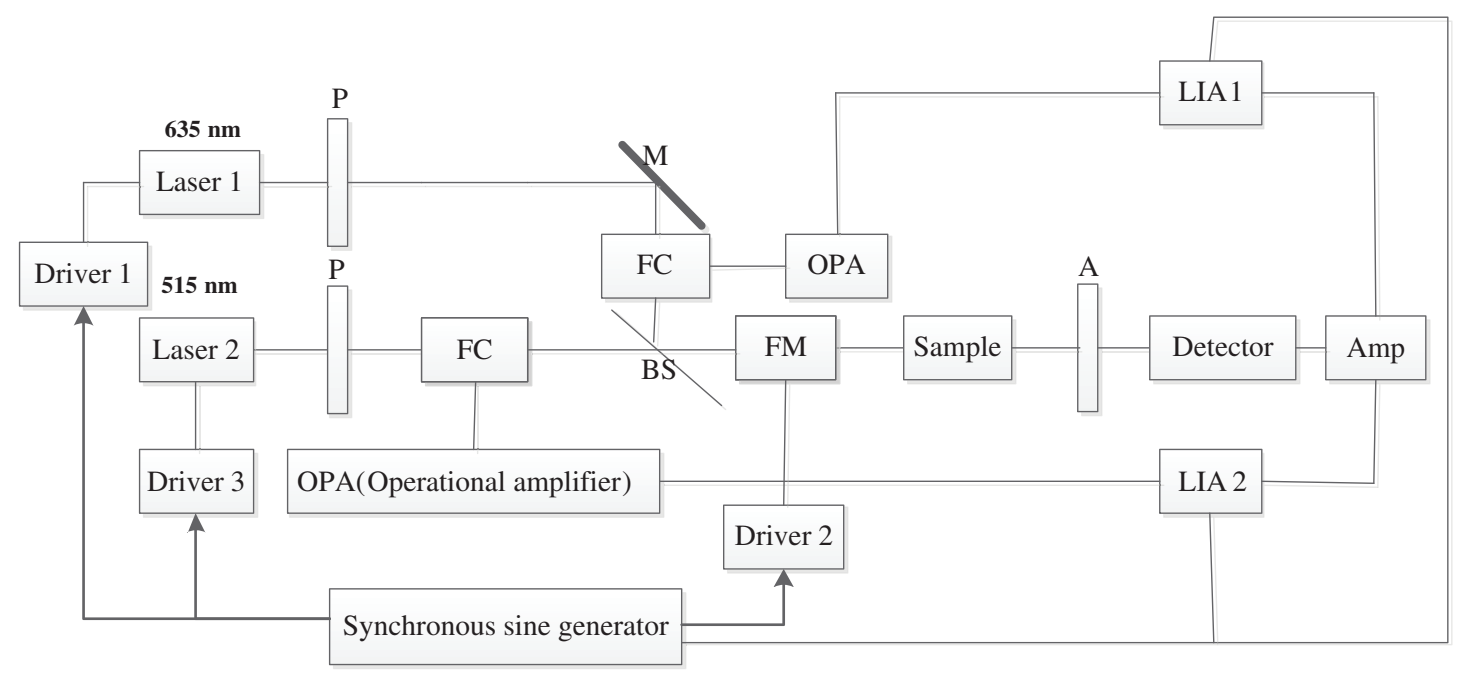

Fig. 1 Block diagram of dual-modulation, dual-wavelength, optical polarimetry system. 
rotations originating from the combination of the glucose and induced birefringence changes with motion artifact.

The in vitro studies were performed using the birefringent test cell, which was stationary or moved up and down and was filled with glucose-doped water solutions in the physiological range of 0 to $600 \mathrm{mg} / \mathrm{dl}$. All samples were created from a $1000-\mathrm{mg} / \mathrm{dl}$ stock glucose solution, which was made by dissolving $1.0 \mathrm{~g}$ of D-glucose (EM Science) into 100-ml deionized water and was mixed with a magnetic stirrer for $8 \mathrm{~h}$ in order to completely dissolve the solute. The stock solution was left undisturbed for at least $3 \mathrm{~h}$ to allow for mutarotation before being used.

\subsection{Mathematical Description of the System}

Without laser modulation, the intensity detected by the detector for each wavelength in the closed-loop system is derived by Jones vectors and matrices and can be described as ${ }^{27-29}$

$$
I \propto E^{2}=\left(\varphi^{2}+\frac{\theta_{\mathrm{m}}^{2}}{2}\right)+2 \varphi \theta_{\mathrm{m}} \sin \left(2 \pi f_{\mathrm{m}}\right) t-\frac{\theta_{\mathrm{m}}}{2} \cos \left(4 \pi f_{\mathrm{m}}\right) t
$$

where $I$ is the intensity of the detected signal, $\varphi$ is the rotation due to the optically active sample, $\theta_{\mathrm{m}}$ is the modulation depth of the FM, $f_{\mathrm{m}}$ is the modulation frequency from the Faraday rotator, and $t$ is time.

For the dual-modulation, dual-wavelength system, the lasers were modulated by sinusoidal signals at frequencies of $f_{\mathrm{c} 1}=45 \mathrm{kHz}, f_{\mathrm{c} 2}=82 \mathrm{kHz}$, respectively, and were also synchronously modulated by the Faraday rotator at a frequency of $f_{\mathrm{m}}=8.75 \mathrm{kHz}$. Due to the crossed-polarized nature of the alignment and according matrices for the optical components presented in the block diagram in Fig. 1, the detected signal of each wavelength can be represented as

$$
\begin{aligned}
I_{i} \propto & V_{\mathrm{DC} i}\left(\varphi_{i}^{2}+\frac{\theta_{\mathrm{m}}^{2}}{2}\right)+\left(\varphi_{i}^{2}+\frac{\theta_{\mathrm{m}}^{2}}{2}\right) V_{\mathrm{c} i} \sin 2 \pi f_{\mathrm{c} i} t \\
& +\varphi_{i} \theta_{\mathrm{m}} V_{\mathrm{c} i} \cos 2 \pi\left(f_{\mathrm{c} i}-f_{\mathrm{m}}\right) t-\varphi_{i} \theta_{\mathrm{m}} V_{\mathrm{c} i} \cos 2 \pi\left(f_{\mathrm{c} i}+f_{\mathrm{m}}\right) t \\
& -\frac{\theta_{\mathrm{m}}^{2} V_{\mathrm{c} i}}{4} \sin 2 \pi\left(f_{\mathrm{c} i}-2 f_{\mathrm{m}}\right) t-\frac{\theta_{\mathrm{m}}^{2} V_{\mathrm{c} i}}{4} \sin 2 \pi\left(f_{\mathrm{c} i}+2 f_{\mathrm{m}}\right) t \\
& +2 V_{\mathrm{DC} i} \varphi_{i} \theta_{\mathrm{m}} \sin \left(2 \pi f_{\mathrm{m}} t\right)-\frac{V_{\mathrm{DC} i} \theta_{\mathrm{m}}^{2}}{2} \cos \left(4 \pi f_{\mathrm{m}} t\right)
\end{aligned}
$$

(see Appendix for derivation) where $V_{\mathrm{c} i}$ represents the amplitude of the sinusoidal signal at each wavelength, $f_{\mathrm{c} i}$ is the carrier frequency of each of the laser modulation signals, and the subscript, $i$, is either 1 or 2 , depending on whether it is the first or second laser modulation wavelength. Thus, the major components of the detected signal at each wavelength include $f_{\mathrm{c} 1}, f_{\mathrm{c} 2}, 2 f_{\mathrm{m}}, f_{\mathrm{c} 1} \pm 2 f_{\mathrm{m}}, f_{\mathrm{c} 2} \pm 2 f_{\mathrm{m}}$ and noise. Equation (3) describes the detected intensity of the light from each wavelength at any instance in time.

In the presence of an optical active element such as glucose, each wavelength of light experiences rotation, $\varphi_{i}$, which is a constant and depending on the concentration of sample and the wavelength of light used, a different sideband frequency component $f_{\mathrm{c} 1} \pm f_{\mathrm{m}}, f_{\mathrm{c} 2} \pm f_{\mathrm{m}}$ was observed, which had a proportional increase with the increase in the rotation, $\varphi_{i}$. Two lock-in amplifiers were used to lock into the detected signals at the frequency of interest, namely, $f_{\mathrm{c} 1}+f_{\mathrm{m}}$ and $f_{\mathrm{c} 2}-f_{\mathrm{m}}$ to extract the information carried by each wavelength, which in turn was processed to obtain the concentration of the glucose in the sample. Since the lock-in amplifiers locked in to the sum of the modulation frequency and one of the carrier frequencies, phase-sensitive detection was used. In order to measure the amplitude of the signals at a specified reference frequency, the frequency and phase stability had to be maintained to prevent signal drift, and hence a synchronous sinusoidal signal generator programmed in LabVIEW 10.0 and implemented through PCI-6713 was used to generate the carrier signals, modulation signal, and reference signals.

\subsection{Theoretical Description for Overcoming Motion Artifact}

Mathematically, as noted above, the operation of each of the single wavelengths is based on Eq. (3). From Eq. (3), it is evident that with any optically active sample the signal has DC terms and, at each wavelength, has major frequency components at $f_{\mathrm{c} 1}, f_{\mathrm{c} 2}, 2 f_{\mathrm{m}}, f_{\mathrm{c} 1} \pm 2 f_{\mathrm{m}}, f_{\mathrm{c} 2} \pm 2 f_{\mathrm{m}}$ and without an optically active sample there are no components at $f_{\mathrm{c} 1} \pm f_{\mathrm{m}}, f_{\mathrm{c} 2} \pm f_{\mathrm{m}}$, because the rotation, $\varphi_{i}$, due to glucose is zero. So when an optically active element such as glucose is present the rotation, $\varphi_{i}$, will cause the intensity of detected signal to vary at the frequencies of $f_{\mathrm{c} 1} \pm f_{\mathrm{m}}, f_{\mathrm{c} 2} \pm f_{\mathrm{m}}$ and the signals at these frequencies proportionally increase with the increase in the rotation, $\varphi_{i}$, depending on the concentration of sample and the wavelength ( $i=1$ or $i=2$ ) of light used. So locking into one of sideband frequency signals provides glucose concentration information carried by each wavelength.

In a closed-loop system, if there is minimal birefringence (i.e., not enough to make the polarization become circular) and the birefringence of the sensing site did not change, the glucose concentration could be extracted from the feedback voltage to the FCs from either wavelength signal, since each compensates for the rotation in polarization due to glucose. In this case, $\varphi_{\mathrm{g} i}$, (rotation at wavelength $i$ due to glucose) is equal to $\varphi_{\mathrm{f} i}$ (rotation at wavelength $i$ fed back to the FC), although the rotation is different for each wavelength and follows Drudes law. However, when motion artifact is introduced into the system, $\varphi_{i}$ becomes a function of birefringence, glucose, and the feedback of the Faraday for a given wavelength: $\varphi_{i}=\varphi_{\mathrm{g} i}+\varphi_{\mathrm{b} i}-\varphi_{\mathrm{f} i}$, where, $\varphi_{\mathrm{b} i}$ is the rotation induced by the birefringence from each wavelength, but the relationship between $\varphi_{\mathrm{b} 1}$ and $\varphi_{\mathrm{b} 2}$ are fixed as long as the wavelengths are known because birefringence is a function of both the wavelength and birefringence $\left|n_{e}-n_{o}\right|$ for a fixed path length through sample. Birefringence for an individual wavelength can be expressed as $\varphi_{\mathrm{b}, i}(t)=\frac{2 \pi}{\lambda_{i}}\left|n_{e}-n_{o}\right| L(t)$, which is constant with wavelength at a given position, a behavior attributed to the form birefringence of the cornea. ${ }^{34-36}$

For the case of minimal, nonvarying birefringence (without motion), the relation between the feedback voltage and glucose concentration is linear, a linear regression is then used to predicted glucose concentrations, and a predicted concentration model for individual wavelength takes the form: Glucose $\mathrm{pred}=$ slope $*[V(t)]+b$, where $V(t)$ is the compensation voltage signal applied to the FC for each wavelength used to null the detected signal via a closed-loop PID controller, Glucose ${ }_{\text {pred }}$ is the predicted concentration of the sample, slope and $b$ are the slope and intercept of calibration line determined by the regression model for each wavelength. As stated, since the rotation due to glucose varies with wavelength following Drudes 
equation, the values for each are not identical. For the dualwavelength system, a multiple regression model was used to determine the glucose concentration and the model had the form: Glucose pred $=$ slope $_{1} *\left[V_{1}(t)\right]+$ slope $_{2} *\left[V_{2}(t)\right]+b$, where $V_{1}(t)$ and $V_{2}(t)$ are the feedback voltage obtained to nullify the system for the corresponding dual-wavelength system and slope $_{1}$, slope $e_{2}$, and $b$ are the calibration coefficients calculated by multiple linear regression (MLR) model..$^{30,31}$ This MLR model has been shown previously to minimize the effect of time-varying birefringence caused by motion using the dual-wavelength polarimetric system due to the difference in the signal from glucose rotation with wavelength (Drudes equation) and the relative common mode noise in each wavelength from time-varying birefringence.

\section{Results and Discussion}

A series of experiments was performed on the new dual-modulation, dual-wavelength system in order to assess its time response and determine its sensitivity in vitro across the physiological range of glucose both without and with the presence of time-varying birefringence.

\subsection{Speed Determination of the System}

As mentioned earlier, a previous attempt to minimize the effect of time-varying corneal birefringence in the eye that used a dualwavelength system was only modulated at $1.09 \mathrm{kHz}$ and required $100 \mathrm{~ms}$ time constant setting on the lock-in amplifiers, resulting in around $300 \mathrm{~ms}$ response time to stabilize the system. ${ }^{29}$ With our new system the time response was measured by visualizing the response speed of the feedback voltage produced by the control system. One widely used measure of the speed of response is the $10 \%$ to $90 \%$ rise time or the amount of time the system takes to go from $10 \%$ to $90 \%$ of the steady-state. For the response speed studies, $300 \mu$ s was the lock-in amplifier time constant that was used. In Fig. 2, the feedback voltages as a function of time for the single wavelength systems (515 and $635 \mathrm{~nm}$, respectively) are depicted. The plot indicates that the PID control system can reach stability in less than $10 \mathrm{~ms}$, which is $\sim 30$ times faster compared with the real-time, dualwavelength approach presented by Malik and Coté. ${ }^{29}$ This

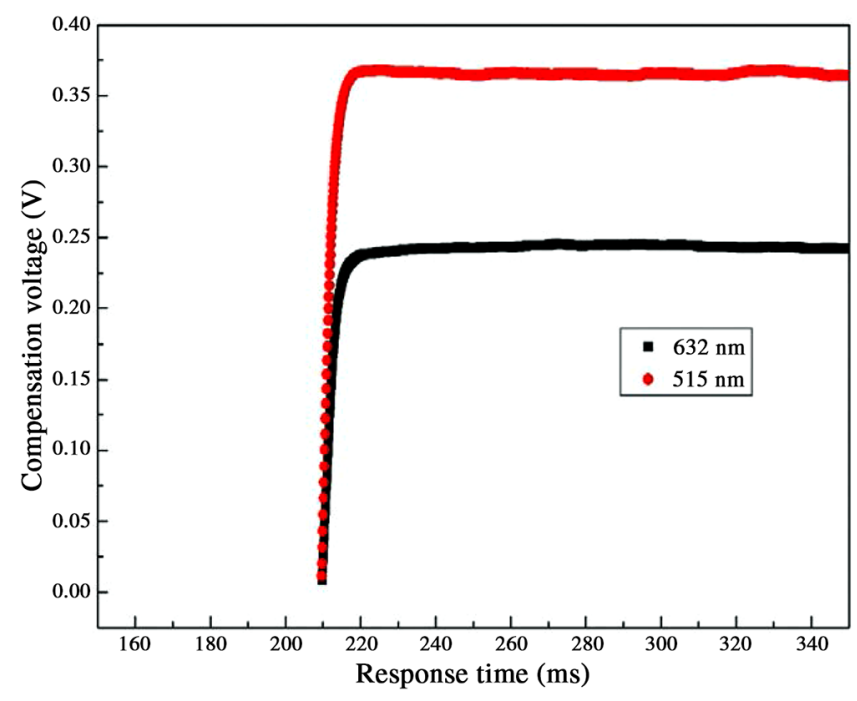

Fig. 2 Stabilization of 515 and $635 \mathrm{~nm}$ PID control system without motion. shows that the laser intensity modulation combined with Faraday modulation and an adaptive PID controller can improve the response speed of the system.

\subsection{System Performance without Motion}

Although it has been shown in the previous section that the new system can provide enhancement in speed, the performance in terms of sensitivity to glucose measurements over the same range as shown previously but requiring less time needs to be determined. The performance of the system was first tested and evaluated for glucose-doped water solutions in the motionless system. In particular, a 1-cm rectangular quartz cuvette that allowed for sample to be easily pipetted into was mounted in a fixed holder. The experiments were performed for individual glucose samples between the concentration range of 0 to $600 \mathrm{mg} / \mathrm{dl}$. The sample cell was in a fixed position for each sample in the motionless system.

The motionless system was calibrated, and then the glucose concentrations were calculated using a simple linear regression model of the data acquired without motion. ${ }^{37}$ In each experiment, three runs of data were recorded for the two wavelengths, 635 and $515 \mathrm{~nm}$, respectively. As it was shown in Sec. 2, the relation between the measured signal in volts and sample concentration is linear, so least-squares linear regression was used for the validation of the model. The predicted versus actual glucose concentrations were plotted for the single wavelength system as shown in Figs. 3 and 4, respectively. It can be observed that the plots indicate the dependency of glucose on one wavelength only; the slopes of this fitted line are slope $1=2591.12$, slope $2=2605.89$, slope $3=2574.22$ for the green $(515 \mathrm{~nm})$ light and for the red $(635 \mathrm{~nm})$ light are slope1 $=2609.30$, slope $2=2570.36$, slope $3=2598.10$, which remained relatively constant. One can see that a high degree of linearity exists given the correlation coefficients for the green $(515 \mathrm{~nm})$ light are $r_{1}=0.9990, r_{2}=0.9993, r_{3}=0.9993$ and for the red $(635 \mathrm{~nm})$ light are $r_{1}=0.9985, r_{2}=0.9993, r_{3}=0.9990$, respectively. Further, the average standard error of prediction (SEP) for the validation of the glucose-doped water was $6.48 \mathrm{mg} / \mathrm{dl}$ for the green light and $7.18 \mathrm{mg} / \mathrm{dl}$ for the red light. The feedback voltages for both wavelengths were applied

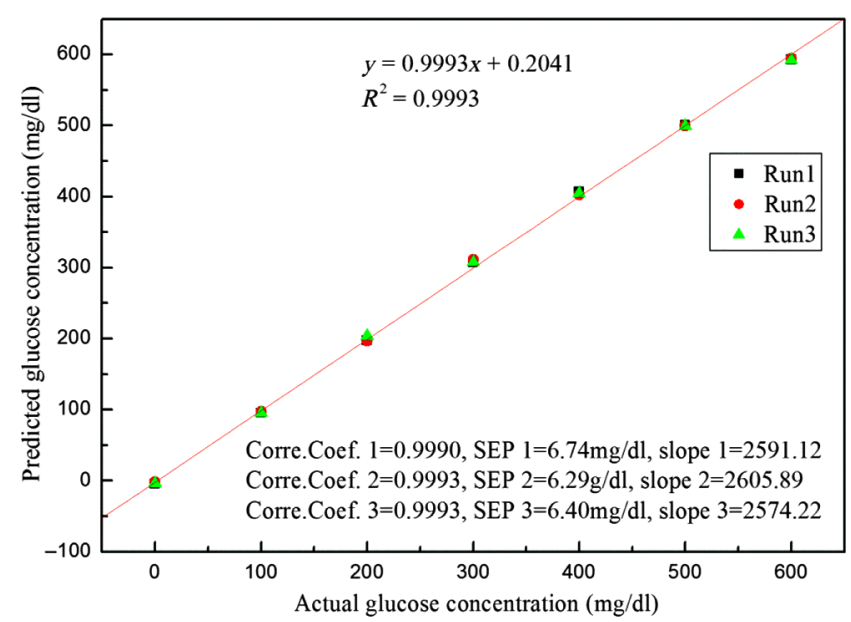

Fig. 3 Predicted glucose concentration as a function of actual glucose concentration for the 515-nm laser without motion using a single linear regression model. 


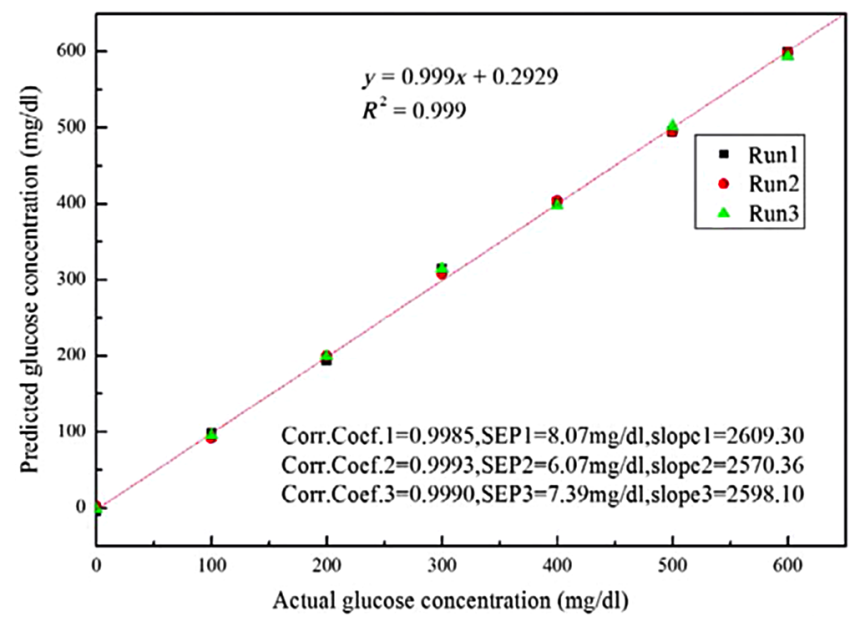

Fig. 4 Predicted glucose concentration as a function of actual glucose concentration for the 635-nm laser wavelength without motion using a single linear regression model.

in an MLR model, which was used to predict the glucose concentration, and Fig. 5 shows the estimated glucose concentration as a function of the actual glucose concentration when both wavelengths are taken into account. The slopes of MLR model are slope $11=-2091.5$, slope $12=748.47$, slope $21=-1463.52$, slope $22=1664.27$, slope $31=-1703.72$, and slope $32=$ 1362.41 for the different runs. Using the MLR model, the information from both wavelengths was combined to improve the results without motion and indeed one can see that a high degree of linearity still exists for all three cases since each correlation coefficient exceeds 0.9996 and the SEP is slightly less than using each wavelength alone ranging from 4.23 to $5.63 \mathrm{mg} / \mathrm{dl}$, with no outliers present in any of three data runs.

\subsection{System Performance with Motion from Time-Varying Birefringence}

The experiments were conducted to test the performance of the system for glucose solution in the sample cell while introducing motion into the system. For these experiments, the test cell was mounted on a programmable translation stage (Thorlabs,

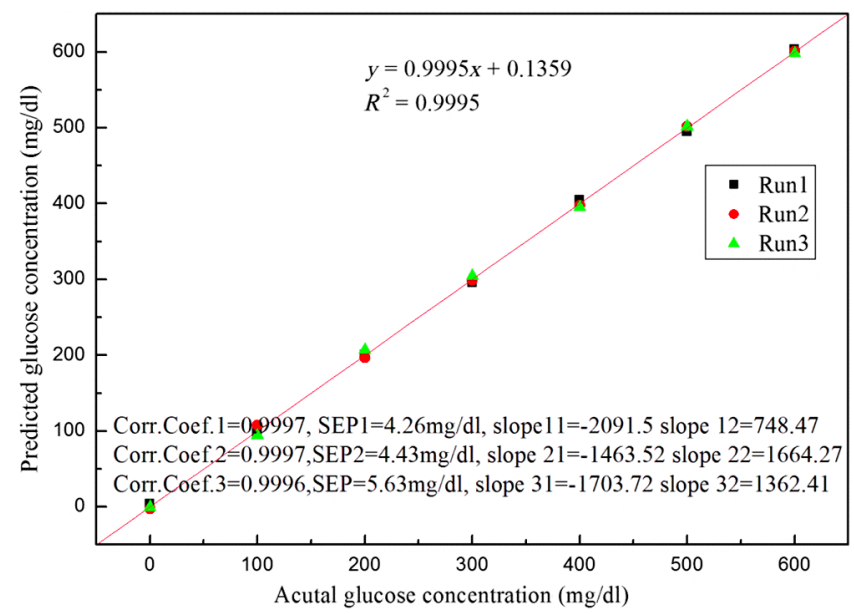

Fig. 5 Predicted glucose concentration as a function of actual glucose concentration for the combined 635 and $515 \mathrm{~nm}$ laser wavelengths without motion using an MLR model.

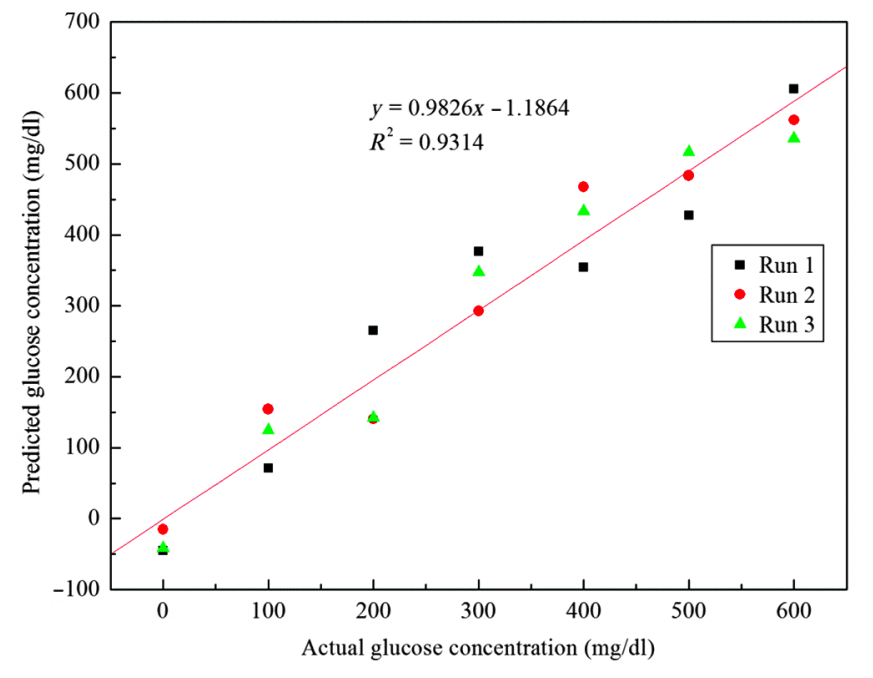

Fig. 6 Actual versus predicted glucose concentration for glucosedoped water in the presence of time varying birefringence due to motion using a single wavelength closed-loop system for $515 \mathrm{~nm}$.

Newtown, New Jersey) and the stage was controlled by a T-Cube DC Servo Motor Controller (Thorlabs, Newtown, New Jersey) and allowed to move up and down to simulate the birefringence changes of the cornea due to motion artifact. The speed and distance of the translation of the motor stage were controlled by a visual basic program.

The system with motion was calibrated and the glucose concentrations were extracted using a linear regression model. This was done for glucose concentrations from 0 to $600 \mathrm{mg} / \mathrm{dl}$.

In order to compare the results of the single wavelength closed-loop and dual-wavelength closed-loop system, actual versus predicted glucose concentration for glucose-doped water coupled with a varying birefringence signal using the single wavelength closed-loop system for each wavelength (515 and $635 \mathrm{~nm}$ ) is plotted in Figs. 6 and 7. As shown in Figs. 6 and 7 , the glucose concentrations cannot be predicted very well using a single closed-loop system, since the change in the birefringence of the sample masks the optical rotation

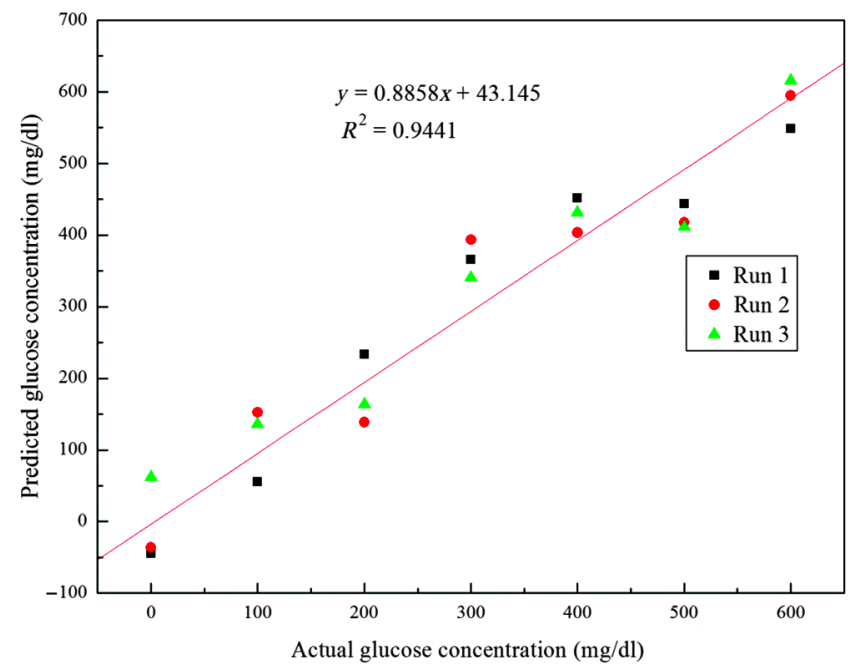

Fig. 7 Actual versus predicted glucose concentration for glucosedoped water in the presence of time varying birefringence due to motion using a single wavelength closed-loop system for $635 \mathrm{~nm}$. 


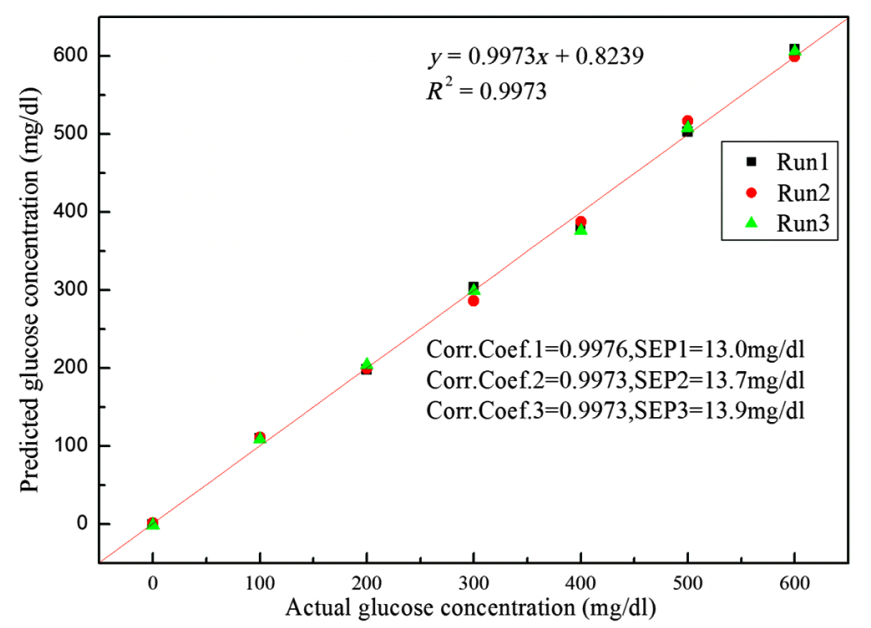

Fig. 8 Predicted glucose concentration as a function of actual glucose concentration with motion due to time-varying birefringence calculated using an MLR regression model for 635 and $515 \mathrm{~nm}$ wavelengths.

due to glucose in the sample. Thus, the single wavelength model has an SEP over $100 \mathrm{mg} / \mathrm{dl}$ in the presence of birefringence changes due to motion.

The predicted concentrations results for the dual-wavelength experiments using MLR calibration are shown in Fig. 8. The predicted calibration results when both wavelengths are used to compensate for the correlated noise due to motion showed a high degree of linearity with all correlation coefficients exceeding 0.9972 and the standard errors of prediction of 13.0, 13.7, and $13.9 \mathrm{mg} / \mathrm{dl}$ as shown in Fig. 8. No outliers were present in any of the data sets. Although the SEP was greater than the motionless system, it was still as good as the previous slower system, and a very simple sample was still within the range needed for noninvasive glucose monitoring.

\section{Conclusions}

In summary, a dual-modulation, dual-wavelength, near real-time closed-loop polarimeter system for glucose monitoring was presented that utilized the combination of laser modulation and Faraday polarization modulation for each wavelength simultaneously. This approach was shown to increase the speed over previous systems by 30 times with a stability of less than $10 \mathrm{~ms}$. Further, the system complexity was reduced by removing one detector from the previous system configuration. Lastly, the in vitro glucose measurement results with and without motion demonstrate the SEP of the system to be within 8.07 and $13.9 \mathrm{mg} / \mathrm{dl}$, respectively, indicating equivalent sensitivity to the previous slower systems in overcoming the noise due to motion artifact with less complexity, showing the potential for its utility as a noninvasive glucose monitoring system. In the future, the steps needed to show this approach has clinical viability are to first perform human subject tests to assess the motion artifact is removed in vivo with light that can be repeatedly coupled through the eye within and between subjects without the use of an external coupler and, second, to assess the potential time delay from blood to aqueous humor in a human.

\section{Acknowledgments}

The authors wish to acknowledge the financial support from the program of China Scholarships Council (CSC). The authors would like to thank Danial Grundan, former MS student in the Optical Biosensing Laboratory at Texas A\&M, and Qiu Qi, professor of School of Photoelectronic Information at the University of Electronic Science and Technology of China, for their assistance and helpful technical discussions. As per SPIE guidelines, the authors acknowledge the SPIE Photonics West proceedings number 9715-29, in which part of this work was presented in San Francisco, California, on February 16, 2016.

\section{Appendix: Appendix for Eq. (3)}

The use of normalized Jones vectors and matrices provides a convenient mechanism to model the detected optical signal. Each matrix represents the operation that a specific optical component performs on the electrical field vector. The system of matrices that represents the optical train is shown in Fig. 1

$$
\begin{aligned}
& \vec{E}=\left[\begin{array}{l}
E_{x}(t) \\
E_{y}(t)
\end{array}\right]=\left[\begin{array}{ll}
1 & 0 \\
0 & 0
\end{array}\right] *\left[\begin{array}{cc}
\cos \left(\varphi_{\mathrm{f}}\right) & \sin \left(\varphi_{\mathrm{f}}\right) \\
-\sin \left(\varphi_{\mathrm{f}}\right) & \cos \left(\varphi_{\mathrm{f}}\right)
\end{array}\right] \\
& *\left[\begin{array}{cc}
\cos \left(\varphi_{\mathrm{g}}\right) & -\sin \left(\varphi_{\mathrm{g}}\right) \\
\sin \left(\varphi_{\mathrm{g}}\right) & \cos \left(\varphi_{\mathrm{g}}\right)
\end{array}\right] \\
& *\left[\begin{array}{cc}
\cos \left[\theta_{\mathrm{m}} \sin \left(2 \pi f_{\mathrm{m}} t\right)\right] & -\sin \left[\theta_{\mathrm{m}} \sin \left(2 \pi f_{\mathrm{m}} t\right)\right] \\
\sin \left[\theta_{\mathrm{m}} \sin \left(2 \pi f_{\mathrm{m}} t\right)\right] & \cos \left[\theta_{\mathrm{m}} \sin \left(2 \pi f_{\mathrm{m}} t\right)\right]
\end{array}\right] \\
& *\left[\begin{array}{ll}
0 & 0 \\
0 & 1
\end{array}\right]\left[\begin{array}{c}
0 \\
\sqrt{V_{\mathrm{DC}}+V_{\mathrm{c}} \sin 2 \pi f_{\mathrm{c}} t}
\end{array}\right] \\
& =\left[\begin{array}{c}
-\sqrt{\mathrm{V}_{\mathrm{DC}}+V_{\mathrm{c}} \sin 2 \pi f_{\mathrm{c}} t} * \sin \left[\theta_{\mathrm{m}} \sin \left(2 \pi f_{\mathrm{m}} t\right)+\varphi_{\mathrm{g}}-\varphi_{\mathrm{f}}\right] \\
0
\end{array}\right] \text {. }
\end{aligned}
$$

The intensity $(I)$ on the photo-detector at any instance in time is proportional to the square of the electric field. If we assume that $\sin \left(\theta_{\mathrm{m}} \sin \left(2 \pi f_{\mathrm{m}} t\right)\right) \approx \theta_{\mathrm{m}} \sin \left(2 \pi f_{\mathrm{m}} t\right)$ for $\theta_{\mathrm{m}} \sin \left(2 \pi f_{\mathrm{m}} t\right) \ll 1$, then the detected intensity can be represented as

$$
\begin{aligned}
& I \propto E^{2} \\
&=\left\{-\sqrt{\mathrm{V}_{\mathrm{DC}}+V_{\mathrm{c}} \sin 2 \pi f_{\mathrm{c}}} t * \sin \left[\theta_{\mathrm{m}} \sin \left(2 \pi f_{\mathrm{m}} t\right)+\varphi_{\mathrm{g}}-\varphi_{\mathrm{f}}\right]\right\}^{2} \\
&=\left(\mathrm{V}_{\mathrm{DC}}+V_{\mathrm{c}} \sin 2 \pi f_{\mathrm{c}} t\right) *\left\{\sin \left[\theta_{\mathrm{m}} \sin \left(2 \pi f_{\mathrm{m}} t\right)+\varphi_{\mathrm{g}}-\varphi_{\mathrm{f}}\right]\right\}^{2} \\
&=\left(\mathrm{V}_{\mathrm{DC}}+V_{\mathrm{c}} \sin 2 \pi f_{\mathrm{c}} t\right) *\left\{\theta_{\mathrm{m}} \sin \left(2 \pi f_{\mathrm{m}} t\right)+\varphi\right\}^{2} \\
&=\left(\mathrm{V}_{\mathrm{DC}}+V_{\mathrm{c}} \sin 2 \pi f_{c} t\right) \\
& *\left\{\theta_{\mathrm{m}}^{2} \sin ^{2}\left(2 \pi f_{\mathrm{m}} t\right)+2 \varphi \theta_{\mathrm{m}} \sin \left(2 \pi f_{\mathrm{m}} t\right)+\varphi^{2}\right\} \\
&=\left(\mathrm{V}_{\mathrm{DC}}+V_{\mathrm{c}} \sin 2 \pi f_{\mathrm{c}} t\right) \\
& *\left\{\theta_{\mathrm{m}}^{2} \frac{\left[1-\cos \left(2 * 2 \pi f_{\mathrm{m}} t\right)\right]}{2}+2 \varphi \theta_{\mathrm{m}} \sin \left(2 \pi f_{\mathrm{m}} t\right)+\varphi^{2}\right\} \\
&= V_{\mathrm{DC}}\left(\varphi^{2}+\frac{\theta_{\mathrm{m}}^{2}}{2}\right)+\left(\varphi^{2}+\frac{\theta_{\mathrm{m}}^{2}}{2}\right) V_{\mathrm{c}} \sin 2 \pi f_{\mathrm{c}} t \\
&+\varphi \theta_{\mathrm{m}} V_{\mathrm{c}} \cos 2 \pi\left(f_{\mathrm{c}}-f_{\mathrm{m}}\right) t-\varphi \theta_{\mathrm{m}} V_{\mathrm{c}} \cos 2 \pi\left(f_{\mathrm{c}}+f_{\mathrm{m}}\right) t \\
&-\frac{\theta_{\mathrm{m}}^{2} V_{\mathrm{c}}}{4} \sin 2 \pi\left(f_{\mathrm{c}}-2 f_{\mathrm{m}}\right) t-\frac{\theta_{\mathrm{m}}^{2} V_{\mathrm{c}}}{4} \sin 2 \pi\left(f_{\mathrm{c}}+2 f_{\mathrm{m}}\right) t \\
&+2 V_{\mathrm{DC}} \varphi \theta_{\mathrm{m}} \sin \left(2 \pi f_{\mathrm{m}} t\right)-\frac{V_{\mathrm{DC}} \theta_{\mathrm{m}}^{2}}{2} \cos \left(4 \pi f_{\mathrm{m}} t\right) .
\end{aligned}
$$




\section{References}

1. A. Alwan, Global Status Report on Noncommunicable Diseases 2010, World Health Organization, WHO Press, Geneva (2011).

2. Centers for Disease Control and Prevention. National Diabetes Statistics Report: Estimates of Diabetes and Its Burden in the United States, U.S. Department of Health and Human Services, Atlanta, Georgia (2014).

3. T. D. Control and Complications Trial Research Group, "The effect of intensive treatment of diabetes on the development and progression of long-term complications in insulin-dependent diabetes mellitus," N. Engl. J. Med. 329, 977-986 (1993).

4. S. K. Vashist, "Continuous glucose monitoring systems: a review," Diagnostics 3, 385-412 (2013).

5. T. Koschinsky and L. Heinemann, "Sensors for glucose monitoring: technical and clinical aspects," Diabetes Metab. Res. Rev. 17, 113-123 (2001).

6. A. M. Enejder et al., "Raman spectroscopy for noninvasive glucose measurements," J. Biomed. Opt. 10(3), 031114 (2005).

7. M. J. Scholtes-Timmerman et al., "Raman spectroscopy as a promising tool for noninvasive point-of-care glucose monitoring," J. Diabetes Sci. Technol. 8(5), 974-979 (2014).

8. J. Yadav et al., "Prospects and limitations of non-invasive blood glucose monitoring using near-infrared spectroscopy," Biomed. Signal Process. Control 18, 214-227 (2015).

9. M. Goodarzi et al., "Multivariate calibration of NIR spectroscopic sensors for continuous glucose monitoring," TrAC, Trends Anal. Chem. 67, 147-158 (2015)

10. K. V. Larin et al., "Specificity of noninvasive blood glucose sensing using optical coherence tomography technique: a pilot study," Phys. Med. Biol. 48(10), 1371 (2003).

11. K. V. Larin, M. S. Eledrisi, and M. Motamedi, "Noninvasive blood glucose monitoring with optical coherence tomography," Diabetes Care 25(12), 2263-2267 (2002).

12. H. Ullah et al., "Autocorrelation optical coherence tomography for glucose quantification in blood," Laser Phys. Lett. 12(12), 125602 (2015).

13. M. A. Pleitez et al., "In vivo noninvasive monitoring of glucose concentration in human epidermis by mid-infrared pulsed photoacoustic spectroscopy," Anal. Chem. 85(2), 1013-1020 (2013).

14. J. C. Pickup et al., "Fluorescence based glucose sensors," Biosens. Bioelectron. 20(12), 2555-2565 (2005).

15. J. C. Pickup et al., "In vivo glucose monitoring: the clinical reality and the promise," Biosens. Bioelectron. 20(10), 1897-1902 (2005).

16. J. C. Pickup et al., "Fluorescence based glucose sensors," Biosens. Bioelectron. 20, 2555-2565 (2005).

17. B. Rabinovitch, W. F. March, and R. L. Adams, "Noninvasive glucose monitoring of the aqueous humor of the eye: part I. measurement of very small optical rotation," Diabetes Care 5(3), 254-258 (1982).

18. W. F. March, B. Rabinovitch, and R. L. Adams, "Noninvasive glucose monitoring of the aqueous humor of the eye: part II. Animal studies and the scleral lens," Diabetes Care 5(3), 259-265 (1982).

19. A. Tura, A. Maran, and G. Pacini, "Non-invasive glucose monitoring: assessment of technologies and devices according to quantitative criteria," Diabetes Res. Clin. Pract. 77(1), 16-40 (2007).

20. C. E. Ferrante do Amaral and B. Wolf, "Current development in noninvasive glucose monitoring," Med. Eng. Phys. 30(5), 541-549 (2008).

21. S. K. Vashistr, "Non-invasive glucose monitoring technology in diabetes management: a review," Anal. Chim. Acta 750, 16-27 (2012).

22. J. L. Smith, "The pursuit of noninvasive glucose: 'Hunting the deceitful turkey'," Fourth Edition: Revised and Expanded, copyright 2015, http:// www.mendosa.com/The $\% 20$ Pursuit $\% 20$ of $\% 20$ Noninvsive $\% 20$ Glucose, \%20Fourth\%20Edition.pdf (22 July 2016).

23. E. J. Gillham, "Photoelectric polarimeter using the Faraday effect," Nature 178, 1412-1413 (1956).

24. G. L. Coté, M. D. Fox, and R. B. Northrop, "Noninvasive optical polarimetric glucose sensing using a true phase measurement technique," IEEE Trans. Biomed. Eng. 39(7), 752-756 (1992)

25. M. J. Goetz, Jr., "Microdegree polarimetry for glucose detection," M.S. Thesis, University of Connecticut, Storrs, Connecticut (1992).
26. M. J. Goetz, Jr., M. D. Fox, and R. B. Northrop, "Microdegree polarimetry using a diode laser for glucose detection," in Proc. 18th IEEE Annual Northeast Bioengineering Conf., pp. 97-98, IEEE, New York (1992).

27. B. D. Cameron and G. L. Coté, "Noninvasive glucose sensing utilizing a digital closed-loop polarimetric approach," IEEE Trans. Biomed. Eng. 44, 1221-1227 (1997).

28. Q. Wan, G. L. Coté, and J. B. Dixon, "Dual-wavelength polarimetry for monitoring glucose in the presence of varying birefringence," J. Biomed. Opt. 10(2), 024029 (2005).

29. B. H. Malik and G. L. Coté, "Real-time, closed-loop dual-wavelength optical polarimetry for glucose monitoring," J. Biomed. Opt. 15(1), 017002 (2010).

30. B. H. Malik, C. W. Pirnstill, and G. L. Coté, "Dual wavelength polarimetric glucose sensing in the presence of birefringence and motion artifact using anterior chamber of the eye phantoms," J. Biomed. Opt. 18(1), 017007 (2013).

31. C. W. Pirnstill et al., "In vivo glucose monitoring using dual-wavelength polarimetry to overcome corneal birefringence in the presence of motion," Diabetes Technol. Ther. 14(9), 819-827 (2012).

32. D. T. Grunden, C. W. Pirnstill, and G. L. Coté, "High-speed dual-wavelength optical polarimetry for glucose sensing," Proc. SPIE 8951, 895111 (2014).

33. U.S. Department of Health and Human Services Public Health Service Food and Drug Administration Center for Devices and Radiological Health, Compliance Guide for Laser Products, HHS Publication FDA 86-8260, Food and Drug Administration, Rockville, Maryland (1992).

34. R. Knighton, X.-R. Huang, and L. A. Cavuoto, "Corneal birefringence mapped by scanning laser polarimetry," Opt. Express 16(18), 1373813751 (2008).

35. R. Kingston, "Spectral dependence of corneal birefringence at visible wavelengths," Invest. Ophthalmol. Vis. Sci. 43, 152 (2002).

36. K. Irsch et al., "Modeling and minimizing interference from corneal birefringence in retinal birefringence scanning for foveal fixation detection," Biomed. Opt. Express 2(7), 1955-1968 (2011).

37. C. W. Pirnstill, D. Grunden, and G. L. Coté, "Polarimetric glucose sensing in vitro: a high frequency approach," Proc. SPIE 8591, 859101 (2013).

Zhen Fang $\mathbf{Y u}$ is pursuing her $\mathrm{PhD}$ at the University of Electronic Science and Technology of China, with a focus on the application of polarized light for noninvasive sensing. She was a visiting student in the optical biosensing laboratory within the Department of Biomedical Engineering at Texas A\&M University for two years. During this period she worked on developing an optical polarimetry system for use in noninvasive glucose monitoring. She is a current member of SPIE.

Casey W. Pirnstill is currently employed as Research Biomedical Engineer at Air Force Research Labs Biodynamics Protection Branch at WPAFB. Primary research interests include the use of optics for medical diagnostics, optical imaging, light-tissue interaction modeling/simulation, and biomedical sensing. His research has focused on developing point-of-care devices using lasers, optics/electronics. He is a member of BMES, SPIE, AEMB, member golden key honor society, SAFE Dayton Chapter, and coauthor of 40 publications, proceedings, patents, and abstracts.

Gerard L. Coté is the director for the Center for Remote Health Technologies and Systems and holds the Charles H. \& Bettye Barclay Professorship within the Department of Biomedical Engineering at Texas A\&M University. He is recognized as a worldwide expert in optical sensing for diagnostic and biomedical monitoring applications. $\mathrm{He}$ is a fellow of four societies, coauthor of over 300 publications, proceedings, patents, and abstracts, and is cofounder of four medical device companies. 